\title{
An Integral Equations Method for the Cauchy Problem Connected with the Helmholtz Equation
}

\author{
Yao Sun ${ }^{1}$ and Deyue Zhang $^{2}$ \\ ${ }^{1}$ College of Science, Civil Aviation University of China, Tianjin 300300, China \\ ${ }^{2}$ School of Mathematics, Jilin University, Changchun 130012, China \\ Correspondence should be addressed to Yao Sun; syhf2008@gmail.com
}

Received 13 July 2013; Accepted 12 August 2013

Academic Editor: Evangelos J. Sapountzakis

Copyright (c) 2013 Y. Sun and D. Zhang. This is an open access article distributed under the Creative Commons Attribution License, which permits unrestricted use, distribution, and reproduction in any medium, provided the original work is properly cited.

\begin{abstract}
We are concerned with the Cauchy problem connected with the Helmholtz equation. We propose a numerical method, which is based on the Helmholtz representation, for obtaining an approximate solution to the problem, and then we analyze the convergence and stability with a suitable choice of regularization method. Numerical experiments are also presented to show the effectiveness of our method.
\end{abstract}

\section{Introduction}

The Cauchy problem for the Helmholtz equation arises in many areas of science, such as wave propagation, vibration, and electromagnetic scattering [1-4]. It is well known that the Cauchy problem is unstable. The solution is unique in some proper solution spaces, but it does not depend continuously on the Cauchy data. For the stability of this problem, we can refer to [5-7]. There are many authors in the literature to investigate this problem, and many numerical methods are proposed. In [8], Sun et al. investigate a potential function method for this method based on the Tikhonov regularization. In [4, 9], Marin et al. investigate the boundary element method via alternating iterative and conjugate gradient method. The boundary knot method can be found by Jin and Zheng $[10,11]$. For the method of fundamental solutions, we can refer to Marin and Lesnic [12] and Wei et al. [13]. Study on the moment method and boundary particle method can be found in Wei et al. [14] and Chen and Fu [15].

The main purpose of this paper is to provide a numerical method for solving the Cauchy problem connected with the Helmholtz equation. The main idea is to formulate integral equations to the Cauchy problem by Green's representation theorem for the solution of the Helmholtz equation. This method was used to reconstruct the shape for the Laplace equation, we refer to Cakoni et al. [16, 17], and to solve a Cauchy problem by Chapko and Johansson [18]. In [19], the authors gave a numerical method of the Cauchy problem for the Laplace equation by using single-layer potential function and jump relations and discussed the decay rate for singular values of Laplacian via singular value decomposition.

The outline of this paper is as follows. In Section 2, we present the formulation of integral equations to the Cauchy problem. In Section 3, we solve the integral equations by the Tikhonov regularization method with the Morozov principle and analyze the convergence and stability. Finally, two numerical examples are included to show the effectiveness of our method.

\section{Formulation of Integral Equations}

Let $D \subset \mathbb{R}^{2}$ be a bounded and simply connected domain with a regular boundary $\partial D \in \mathscr{C}^{2}$ and let $\partial D$ consist of two nonintersecting parts $\Gamma$ and $\Sigma, \Sigma \cup \Gamma=\partial D$, where $\Gamma$ and $\Sigma$ are nonempty. In general, we assume that $\Gamma$ is an open-connected subset of $\partial D$. Consider the following Cauchy problem. Given Cauchy data $f_{D}$ and $f_{N}$ on $\Gamma$, we find $u$, such that $u$ satisfies

$$
\begin{gathered}
\Delta u+k^{2} u=0, \quad \text { in } D, \\
u=f_{D}, \quad \text { on } \Gamma, \\
\frac{\partial u}{\partial n}=f_{N}, \quad \text { on } \Gamma,
\end{gathered}
$$


where $n$ is the unit normal to the boundary $\partial D$ directed into the exterior of $D$ and the wave number $k>0$. Without loss of generality, we make the assumption on the measured data that $f_{D} \in H^{1}(\Gamma)$ and $f_{N} \in L^{2}(\Gamma)$ and suppose that the Cauchy problem has a unique solution $u$ in $H^{3 / 2}(D)[14,20]$.

From Green's representation theorem for the solutions of the Helmholtz equation [21], we know that the solution $u$ of (1) has the following form:

$$
\begin{array}{r}
u(x)=\int_{\partial D}\left\{\frac{\partial u}{\partial \nu}(y) \Phi(x, y)-u(y) \frac{\Phi(x, y)}{\partial \nu(y)}\right\} \\
x(y), \\
x \in D .
\end{array}
$$

Here, $\Phi(x, y)=(i / 4) H_{0}^{(1)}(k|x-y|)$.

From the jump relations, we have

$$
\begin{array}{r}
\frac{1}{2} u(x)=\int_{\partial D}\left\{\frac{\partial u}{\partial \nu}(y) \Phi(x, y)-u(y) \frac{\Phi(x, y)}{\partial \nu(y)}\right\} d s(y), \\
x \in \partial D .
\end{array}
$$

Then, we have the following integral equations:

$$
\begin{aligned}
& \int_{\Sigma}\left\{\frac{\partial u}{\partial \nu}(y) \Phi(x, y)-u(y) \frac{\Phi(x, y)}{\partial \nu(y)}\right\} d s(y) \\
&=\frac{1}{2} u(x)-\int_{\Gamma}\left\{\frac{\partial u}{\partial \nu}(y) \Phi(x, y)-u(y) \frac{\Phi(x, y)}{\partial \nu(y)}\right\} d s(y), \\
& x \in \Gamma,
\end{aligned}
$$$$
\int_{\Sigma}\left\{\frac{\partial u}{\partial v}(y) \Phi(x, y)-u(y) \frac{\Phi(x, y)}{\partial \nu(y)}\right\} d s(y)-\frac{1}{2} u(x)
$$$$
=-\int_{\Gamma}\left\{\frac{\partial u}{\partial v}(y) \Phi(x, y)-u(y) \frac{\Phi(x, y)}{\partial v(y)}\right\} d s(y),
$$

$x \in \Sigma$.

Theorem 1. Integral equation (5) has at most one solution.

Proof. It is sufficient to prove that the homogeneous problem has a unique solution $\left(\left.u\right|_{\Sigma},\left.(\partial u / \partial v)\right|_{\Sigma}\right)=(0,0)$, which means that the following equations:

$$
\begin{array}{r}
\int_{\Sigma}\left\{\frac{\partial u}{\partial \nu}(y) \Phi(x, y)-u(y) \frac{\Phi(x, y)}{\partial \nu(y)}\right\} d s(y)=0, \quad x \in \Gamma, \\
\int_{\Sigma}\left\{\frac{\partial u}{\partial \nu}(y) \Phi(x, y)-u(y) \frac{\Phi(x, y)}{\partial \nu(y)}\right\} d s(y)-\frac{1}{2} u(x)=0, \\
x \in \Sigma,
\end{array}
$$

have a unique solution $\left(\left.u\right|_{\Sigma},\left.(\partial u / \partial v)\right|_{\Sigma}\right)=(0,0)$. Let

$$
\begin{array}{r}
\omega(x)=\int_{\Sigma}\left\{\frac{\partial u}{\partial \nu}(y) \Phi(x, y)-u(y) \frac{\Phi(x, y)}{\partial \nu(y)}\right\} d s(y), \\
x \in R^{2} \backslash \partial D .
\end{array}
$$

By (6), we know that $\left.\omega(x)\right|_{\Gamma}=0$. From the properties of single-double layer and the jump relations [22-24], we deduce

$$
\begin{aligned}
\lim _{x \rightarrow \Sigma^{+}} \omega(x)= & \int_{\Sigma}\left\{\frac{\partial u}{\partial v}(y) \Phi(x, y)-u(y) \frac{\Phi(x, y)}{\partial v(y)}\right\} d s(y) \\
& -\frac{1}{2} u(x) .
\end{aligned}
$$

By (7), we know that

$$
\lim _{x \rightarrow \Sigma^{+}} \omega(x)=0,
$$

from the radiation at infinite and the uniqueness of the exterior boundary value problem for the Helmholtz equation yields that $\omega$ vanishes in the exterior of $D$. So $\omega=0$ in $R^{2} \backslash \bar{D}$. Thus, we can easily get

$$
\begin{gathered}
\omega=0, \quad x \in \Gamma, \\
\frac{\partial \omega}{\partial n}=0, \quad x \in \Gamma .
\end{gathered}
$$

$\partial D \in \mathscr{C}^{2}$ yields the uniqueness of the Cauchy problem [7], and we conclude that $\omega=0$ in $R^{2} / \partial D$. From the jump relations [25], we have

$$
\left.u\right|_{\Sigma}=\left.u\right|_{\Sigma^{-}}-\left.u\right|_{\Sigma^{+}}=0,\left.\quad \frac{\partial u}{\partial v}\right|_{\Sigma}=\left.\frac{\partial u}{\partial v}\right|_{\Sigma^{+}}-\left.\frac{\partial u}{\partial v}\right|_{\Sigma^{-}}=0 .
$$

This completes the proof.

For simplicity, we define some operators and symbols as follows:

$$
\begin{array}{ll}
\left(A_{1} \varphi\right)(x)=\int_{\Sigma} \varphi(y) \Phi(x, y) d s(y), & x \in \Gamma, \\
\left(B_{1} \varphi\right)(x)=-\int_{\Sigma} \varphi(y) \frac{\Phi(x, y)}{\partial \nu(y)} d s(y), & x \in \Gamma, \\
\left(A_{2} \varphi\right)(x)=\int_{\Sigma} \varphi(y) \Phi(x, y) d s(y), & x \in \Sigma, \\
\left(B_{2} \varphi\right)(x)=-\int_{\Sigma} \varphi(y) \frac{\Phi(x, y)}{\partial \nu(y)} d s(y), \quad x \in \Sigma,
\end{array}
$$


TABLE 1: Regularization parameter $\alpha$ and errors for Example 1 of Case 1 with $k=3$.

\begin{tabular}{lccc}
\hline Noise & $\alpha$ & $\left\|U_{\alpha(\delta)}^{\delta}-u\right\|_{L^{2}(\Sigma)}$ & $\left\|V_{\alpha(\delta)}^{\delta}-\partial_{n} u\right\|_{L^{2}(\Sigma)} /\left\|\partial_{n} u\right\|_{L^{2}(\Sigma)}$ \\
\hline 0 & $7.78 \times 10^{-15}$ & $8.29 \times 10^{-4}$ & $3.6 \times 10^{-3}$ \\
0.001 & $2.53 \times 10^{-6}$ & $2.20 \times 10^{-2}$ & $5.62 \times 10^{-2}$ \\
0.01 & $2.08 \times 10^{-4}$ & $6.58 \times 10^{-2}$ & $1.21 \times 10^{-1}$ \\
0.03 & $7.75 \times 10^{-4}$ & $8.53 \times 10^{-2}$ & $1.33 \times 10^{-1}$ \\
\hline
\end{tabular}

TABLE 2: Regularization parameter $\alpha$ and errors for Example 1 of Case 1 with $k=8$.

\begin{tabular}{lccc}
\hline Noise & $\alpha$ & $\left\|U_{\alpha(\delta)}^{\delta}-u\right\|_{L^{2}(\Sigma)}$ & $\left\|V_{\alpha(\delta)}^{\delta}-\partial_{n} u\right\|_{L^{2}(\Sigma)} /\left\|\partial_{n} u\right\|_{L^{2}(\Sigma)}$ \\
\hline 0 & $3.36 \times 10^{-16}$ & $9 \times 10^{-3}$ & $4.25 \times 10^{-2}$ \\
0.001 & $1.16 \times 10^{-6}$ & $3.56 \times 10^{-2}$ & $8.07 \times 10^{-2}$ \\
0.01 & $3.14 \times 10^{-5}$ & $5.13 \times 10^{-2}$ & $1.07 \times 10^{-1}$ \\
0.03 & $2.85 \times 10^{-4}$ & $5.24 \times 10^{-2}$ & $1.19 \times 10^{-1}$ \\
\hline
\end{tabular}

$$
\begin{gathered}
f(x)=\frac{1}{2} u(x) \\
-\int_{\Gamma}\left\{\frac{\partial u}{\partial \nu}(y) \Phi(x, y)-u(y) \frac{\Phi(x, y)}{\partial \nu(y)}\right\} d s(y), \\
g(x)=-\int_{\Gamma}\left\{\frac{\partial u}{\partial \nu}(y) \Phi(x, y) \quad-\Gamma,\right. \\
\left.\quad-u(y) \frac{\Phi(x, y)}{\partial \nu(y)}\right\} d s(y), \\
U(x)=\left.u(x)\right|_{\Sigma}, \quad V(x)=\left.\frac{\partial u}{\partial \nu}(x)\right|_{\Sigma}
\end{gathered}
$$

By the above definitions, we have the following simple equations:

$$
\begin{gathered}
\left(A_{1} V\right)(x)+\left(B_{1} U\right)(x)=f(x), \quad x \in \Gamma, \\
\left(A_{2} V\right)(x)+\left(\left(B_{2}-\frac{1}{2} I\right) U\right)(x)=g(x), \quad x \in \Sigma .
\end{gathered}
$$

Supposing that the endpoints of $\Gamma$ are $A$ and $B$, we can find that $v$ satisfies the Helmholtz equation and satisfies $v(A)=$ $u(A), v(B)=u(B)$; let $\omega(x)=u(x)-v(x)$; then $\omega(x)$ is a solution of the Helmholtz equation and $\omega(A)=\omega(B)=0$, so we can fix $f_{D}(A)=f_{D}(B)=0$ and define

$$
\left(A_{2}^{\prime} \psi\right)(x)=\int_{\partial D} \psi(y) \Phi(x, y) d s(y), \quad x \in \Sigma,
$$

where

$$
\psi(y)= \begin{cases}\varphi(y), & y \in \Sigma \\ 0, & y \in \Gamma\end{cases}
$$

Remark 2. For the construction of the function $v$, we can give a simple example. Supposing that $A=(0,0)$ and $B=(1,0)$, $u_{A}=a, u_{B}=b, a \neq b$, we can fix $v(x)=a\left(1-x_{1}\right) e^{i k x_{2}}+b x_{2} e^{i k x_{2}}$.

From zero extension, we will get the following lemma.

Lemma 3. The operator $A_{2}^{\prime}$ is compact from $L^{2}(\partial D)$ to $H^{1}(\partial D)$ [21, Theorem 3.6]; thus, the operators $A_{2}$ and $B_{2}$ are compact from $L^{2}(\Sigma)$ to $L^{2}(\Sigma)$ and $A_{1}$ and $B_{1}$ are compact from $L^{2}(\Sigma)$ to $L^{2}(\Gamma)$.

From Theorem 1, we know that the Cauchy problem has a unique solution without the restriction on $k^{2}$, and thus the homogeneous problem has only trivial solution. With the aid of the jump relations, it can be seen that $B_{2}-(1 / 2) I$ has a trivial null space (for details see [26, Chapter 3.4]). From the Rizes-Fredholm theorem, we can easily get the following theorem.

Theorem 4. The operator $B_{2}-(1 / 2) I$ is bounded invertible.

By the above conclusion, we can get following equations:

$$
\begin{gathered}
{\left[A_{1}-B_{1}\left(B_{2}-\frac{I}{2}\right)^{-1} A_{2}\right] V=f-B_{1}\left(B_{2}-\frac{I}{2}\right)^{-1} g, \quad x \in \Gamma,} \\
U=\left(B_{2}-\frac{I}{2}\right)^{-1}\left(g-A_{2} V\right), \quad x \in \Sigma .
\end{gathered}
$$

To this end, we define the operator $\mathcal{N}: L^{2}(\Sigma) \rightarrow L^{2}(\Gamma)$ by

$$
\mathscr{N} \varphi(x)=\left[A_{1}-B_{1}\left(B_{2}-\frac{I}{2}\right)^{-1} A_{2}\right] \varphi(x) .
$$

Then, the following property of the operator $\mathcal{N}$ holds.

Theorem 5. The operator $\mathcal{N}: L^{2}(\Sigma) \rightarrow L^{2}(\Gamma)$ is compact and injective. 
TABLE 3: Regularization parameter $\alpha$ and errors for Example 2 with $k=5,1 \%$ noise.

\begin{tabular}{lccr}
\hline$\Theta$ & $\alpha$ & $\left\|U_{\alpha(\delta)}^{\delta}-u\right\|_{L^{2}(\Sigma)}$ & $\left\|V_{\alpha(\delta)}^{\delta}-\partial_{n} u\right\|_{L^{2}(\Sigma)} /\left\|\partial_{n} u\right\|_{L^{2}(\Sigma)}$ \\
\hline$\pi / 2$ & $2.02 \times 10^{-2}$ & $1.79 \times 10^{-1}$ & $2.94 \times 10^{-1}$ \\
$\pi$ & $1.03 \times 10^{-4}$ & $5.99 \times 10^{-2}$ & $9.43 \times 10^{-2}$ \\
$3 \pi / 2$ & $3.81 \times 10^{-5}$ & $4.90 \times 10^{-3}$ & $1.96 \times 10^{-2}$ \\
\hline
\end{tabular}

TABLE 4: Regularization parameter $\alpha$ and errors for Example 2 with $k=5,3 \%$ noise.

\begin{tabular}{lccc}
\hline$\Theta$ & $\alpha$ & $\left\|U_{\alpha(\delta)}^{\delta}-u\right\|_{L^{2}(\Sigma)}$ & $\left\|V_{\alpha(\delta)}^{\delta}-\partial_{n} u\right\|_{L^{2}(\Sigma)} /\left\|\partial_{n} u\right\|_{L^{2}(\Sigma)}$ \\
\hline$\pi / 2$ & $4.04 \times 10^{-2}$ & $2.32 \times 10^{-1}$ & $3.13 \times 10^{-1}$ \\
$\pi$ & $3.13 \times 10^{-4}$ & $7.37 \times 10^{-2}$ & $1.19 \times 10^{-1}$ \\
$3 \pi / 2$ & $1.54 \times 10^{-4}$ & $1.83 \times 10^{-2}$ & $5.79 \times 10^{-2}$ \\
\hline
\end{tabular}

Proof. By Lemma 3, we know the operator $\mathcal{N}$ is compact. By Theorems 1 and 4 , we deduce that the operator $\mathcal{N}$ is injective.

Now, we turn to introducing our numerical algorithm. First, function $\phi$ is achieved by solving the following integral equation:

$$
\mathscr{N} V=h(x), \quad x \in \Gamma,
$$

where

$$
h(x)=f-B_{1}\left(B_{2}-\frac{I}{2}\right)^{-1} g, \quad x \in \Gamma .
$$

Remark 6. In general, (19) is not solvable since we cannot assume that the Cauchy data $h$, especially the measured noisy data $h^{\delta}$, are in the range $\mathcal{N}\left(L^{2}(\Gamma)\right)$ of $\mathcal{N}$. Therefore, we will solve (19) by some regularization methods in the next section and then give the error estimates.

\section{Tikhonov Regularization and Morozov Discrepancy Principle}

In this section, we will use the Tikhonov regularization method and the Morozov discrepancy principle to solve the integral system (19) and then give the error estimates and convergence results. In general, we give the noise data $f_{D}^{\delta_{1}}$, $f_{N}^{\delta_{1}}$, and then we should consider the following equations:

$$
\mathcal{N} V^{\delta}=h^{\delta}
$$

Here $h^{\delta} \in L^{2}(\Gamma)$ are measured noisy data satisfying

$$
\left\|h-h^{\delta}\right\|_{L^{2}(\Gamma)} \leq \delta
$$

and it is obvious that $\delta=\mathcal{O}\left(\delta_{1}\right)$.

The Tikhonov regularization of integral system (21) is to solve the following equation:

$$
\alpha V_{\alpha}^{\delta}+\mathcal{N}^{*} \mathcal{N} V_{\alpha}^{\delta}=\mathcal{N}^{*} h^{\delta}
$$

By introducing the regularization operators

$$
R_{\alpha}:=\left(\alpha I+\mathcal{N}^{*} \mathcal{N}\right)^{-1} \mathcal{N}^{*}, \quad \text { for } \alpha>0,
$$

we can achieve the regularized solution $V_{\alpha}^{\delta}=R_{\alpha} h^{\delta}$ of (21). We choose the regularization parameter $\alpha$ by the Morozov discrepancy principle, and then we have the following result.

Theorem 7. Let $\delta$ be sufficiently small positive constant and $\delta<\left\|h^{\delta}\right\|_{L^{2}(\Gamma)}$. Let the Tikhonov solution $V_{\alpha(\delta)}^{\delta}$ satisfy $\left\|\mathcal{N} V_{\alpha(\delta)}^{\delta}-h^{\delta}\right\|_{L^{2}(\Gamma)}=\delta$ for all $\delta \in\left(0, \delta_{0}\right)$ and let $V=\mathcal{N}^{*} z \epsilon$ $\mathcal{N}^{*}\left(L^{2}(\Gamma)\right)$ with $\|z\|_{L^{2}(\Gamma)} \leq E$. Then

$$
\left\|V_{\alpha(\delta)}^{\delta}-V\right\|_{L^{2}(\Sigma)} \leq 2 \sqrt{\delta E}
$$

Here $V \in L^{2}(\Sigma)$ is the exact solution which satisfies (19).

Proof. The statement follows directly from Theorem 2.17 in [25]. lem:

Consider the following Neumann boundary value prob-

$$
\begin{gathered}
\Delta u_{\alpha(\delta)}^{\delta}+k^{2} u_{\alpha(\delta)}^{\delta}=0, \quad \text { in } D \\
\frac{\partial u_{\alpha(\delta)}^{\delta}}{\partial n}=f_{N}^{\delta_{1}}, \quad \text { on } \Gamma, \\
\frac{\partial u_{\alpha(\delta)}^{\delta}}{\partial n}=V_{\alpha}^{\delta}, \quad \text { on } \Sigma
\end{gathered}
$$

where $\delta_{1}=\mathcal{O}(\delta)$, we know that there is a unique weak solution in $H^{1}(D)$ [14].

Then we have the following main result in this paper.

Theorem 8. Let the assumptions in Theorem 7 hold. Then

$$
\left\|u_{\alpha(\delta)}^{\delta}-u\right\|_{H^{1}(D)} \leq C_{1} \delta^{1 / 2}
$$

Moreover, the following estimate on boundary $\Sigma$ holds:

$$
\left\|u_{\alpha(\delta)}^{\delta}-u\right\|_{L^{2}(\Sigma)}+\left\|\frac{\partial u_{\alpha(\delta)}^{\delta}}{\partial n}-\frac{\partial u}{\partial n}\right\|_{L^{2}(\Sigma)} \leq C \delta^{1 / 2}
$$

The positive constant $C$ depends only on $k, D$, and $E$. 


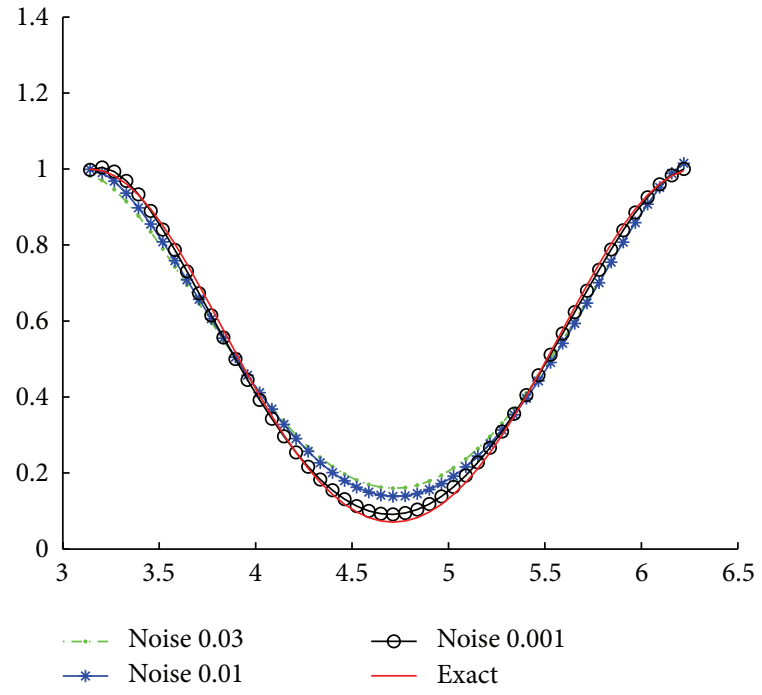

(a) $f$

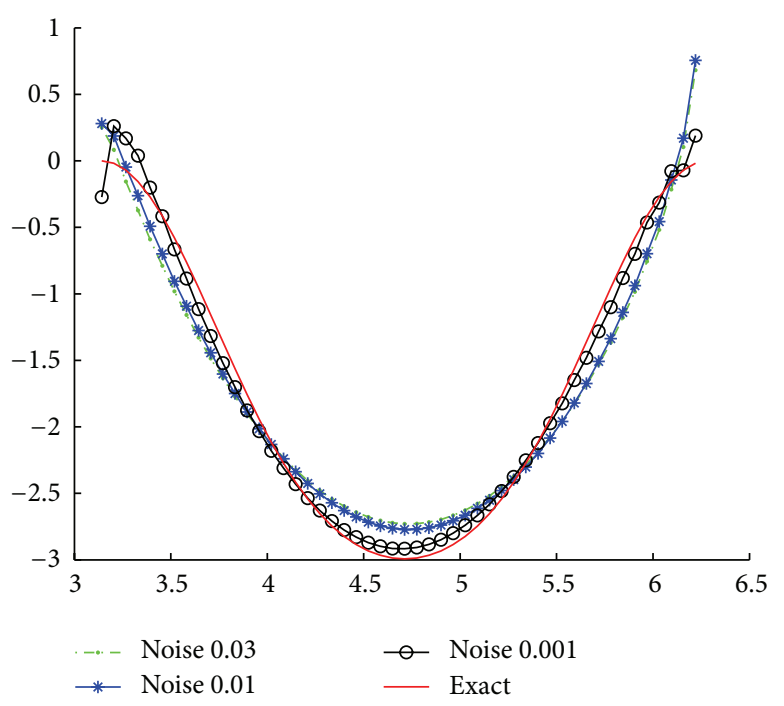

(b) $d$

FIgURE 1: Example 1: the exact solution and the numerical solution on $\Sigma$ with $k=3$ for Case 1.

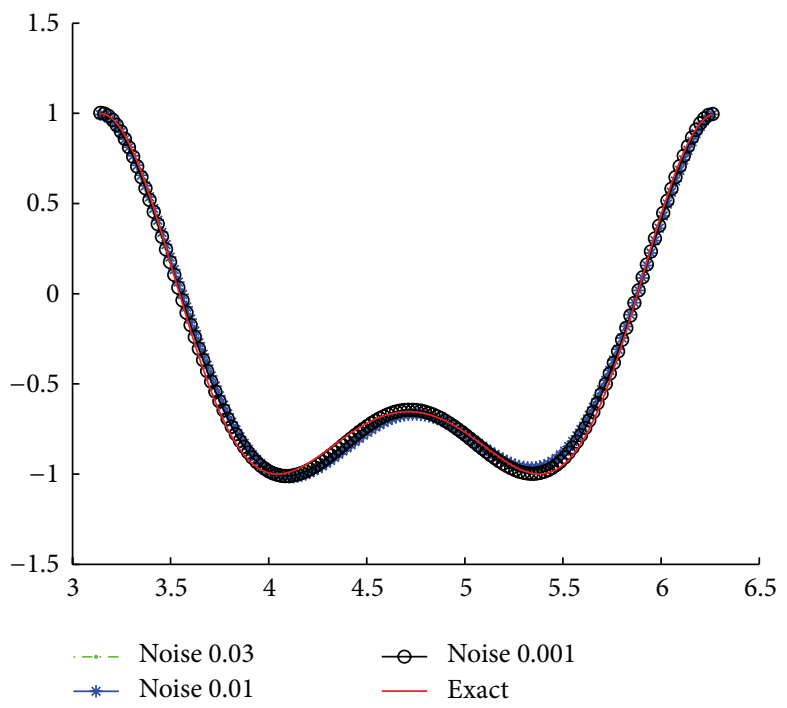

(a) $f$

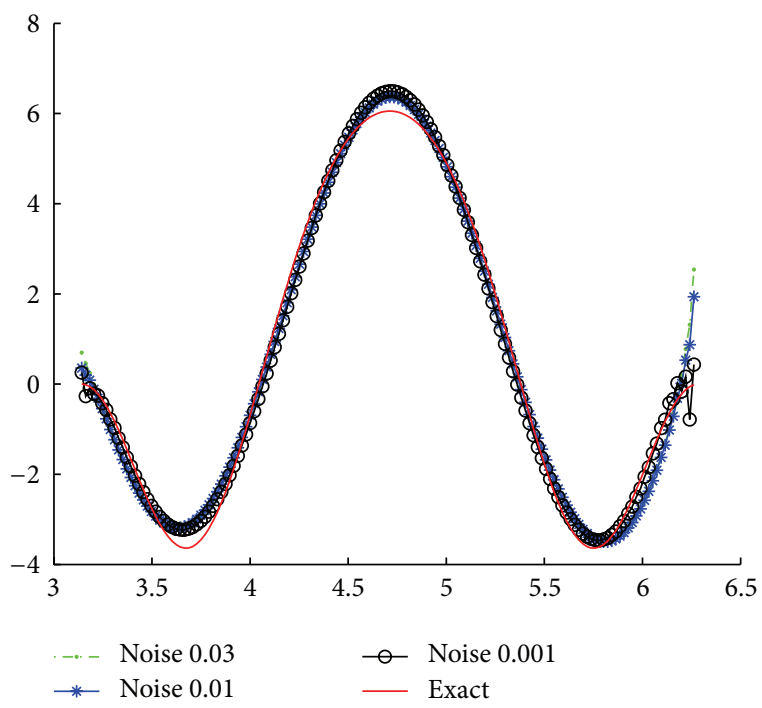

(b) $d$

FIgURE 2: Example 1: the exact solution and the numerical solution on $\Sigma$ with $k=8$ for Case 1 .

Proof. From triangle inequality and Theorem 7, we get

$$
\begin{aligned}
\| u_{\alpha(\delta)}^{\delta}- & u\left\|_{L^{2}(\Sigma)}+\right\| \frac{\partial u_{\alpha(\delta)}^{\delta}}{\partial n}-\frac{\partial u}{\partial n} \|_{L^{2}(\Sigma)} \\
= & \left\|\left(B_{2}-\frac{I}{2}\right)^{-1}\left[\left(g^{\delta}-A_{1} V_{\alpha(\delta)}^{\delta}\right)-\left(g-A_{1} V\right)\right]\right\|_{L^{2}(\Sigma)} \\
& +\left\|V_{\alpha(\delta)}^{\delta}-V\right\|_{L^{2}(\Sigma)} \\
\leq & C_{3}\left\|g^{\delta}-g\right\|_{L^{2}(\Sigma)}+C_{4}\left\|V_{\alpha(\delta)}^{\delta}-V\right\|_{L^{2}(\Sigma)} \leq C \delta^{1 / 2} .
\end{aligned}
$$

The inequalities imply the estimate (28).
From the assumption, we have

$$
\left\|u_{\alpha(\delta)}^{\delta}-u\right\|_{L^{2}(\Gamma)}+\left\|\frac{\partial u_{\alpha(\delta)}^{\delta}}{\partial n}-\frac{\partial u}{\partial n}\right\|_{L^{2}(\Gamma)} \leq 2 \delta_{1} \leq C^{\prime} \delta^{1 / 2} .
$$

Then, we get

$$
\left\|u_{\alpha(\delta)}^{\delta}-u\right\|_{L^{2}(\partial D)}+\left\|\frac{\partial u_{\alpha(\delta)}^{\delta}}{\partial n}-\frac{\partial u}{\partial n}\right\|_{L^{2}(\partial D)} \leq C^{\prime \prime} \delta^{1 / 2} .
$$

The trace theorem and the triangle inequality yield the estimate (27). 


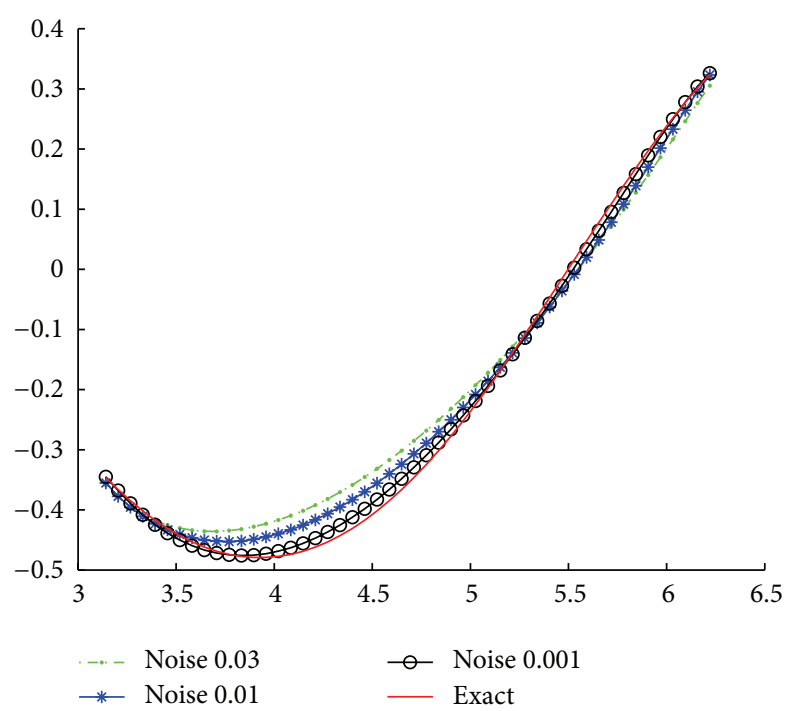

(a) $f$

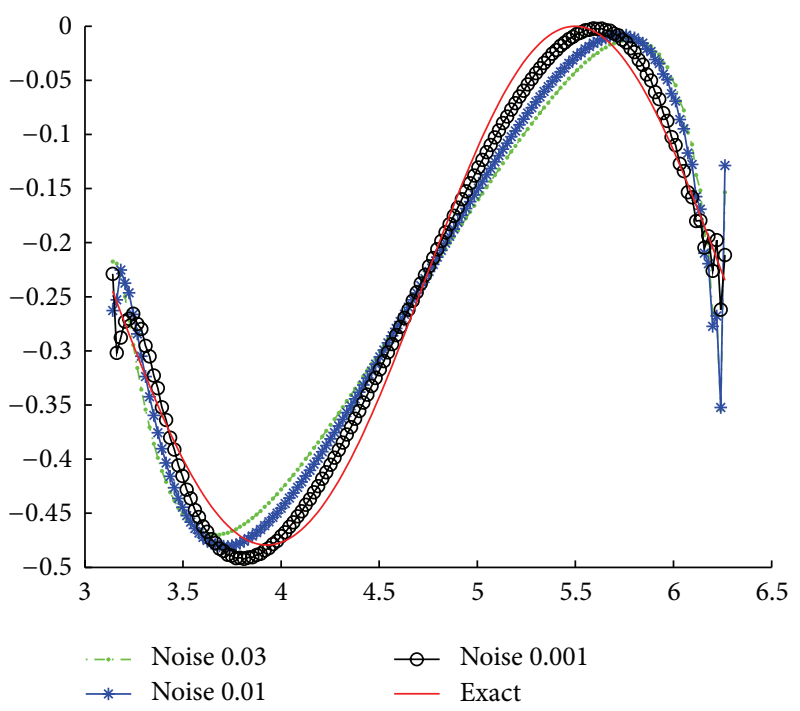

(b) $d$

Figure 3: Example 1: the exact solution and the numerical solution on $\Sigma$ with $k=1$ for Case 2.

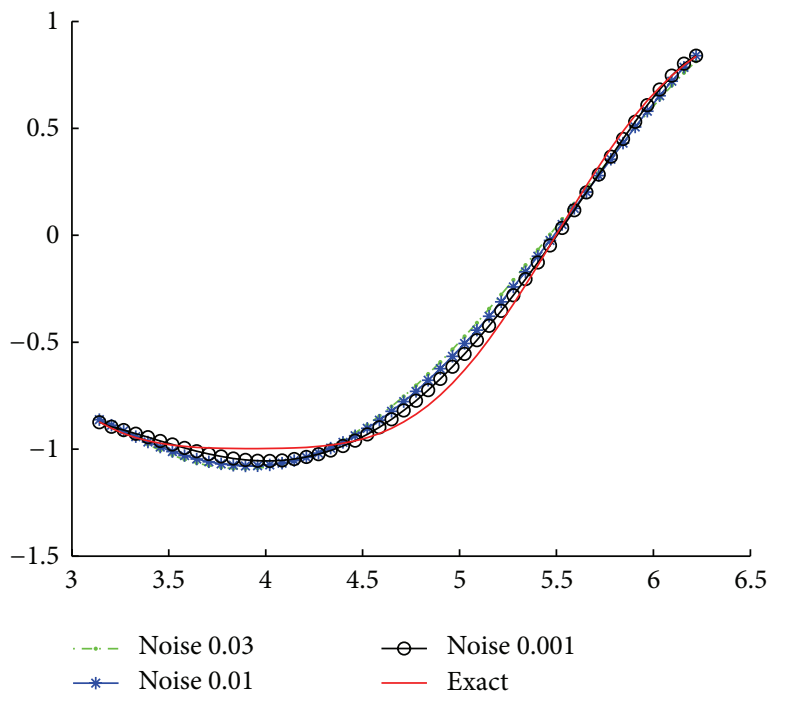

(a) $f$

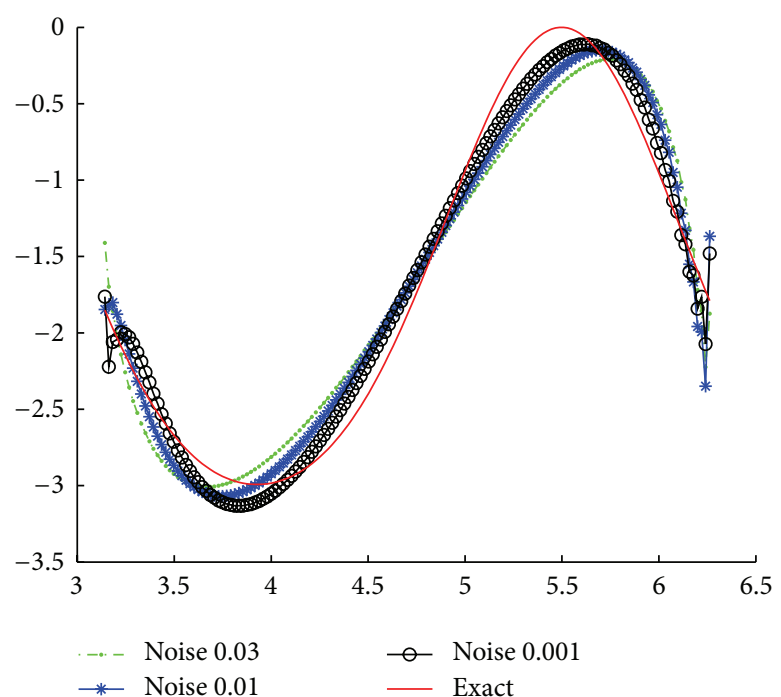

(b) $d$

FIgURE 4: Example 1: the exact solution and the numerical solution on $\Sigma$ with $k=3$ for Case 2.

\section{Numerical Examples}

In this section, we report two examples of $\mathbb{R}^{2}$ to test the effectiveness of our method. In the figures, we denote by $f$ and $d$ the function values and the normal derivative values, respectively. For the discrete of the integral equations, we use the Nyström method, see [23, Chapter 3.5].

Example 1. To test our code, consider the case in which the exact solution to the Cauchy problem is $u(x)=e^{i k x \cdot d}$. Let $D=\left\{\left(x_{1}, x_{2}\right) \mid x_{1}^{2}+x_{2}^{2}<0.5^{2}\right\}$, let $\Gamma=\left\{\left(x_{1}, x_{2}\right) \mid x_{1}^{2}+x_{2}^{2}=\right.$ $\left.0.5^{2}, x_{2} \geq 0\right\}$, and let $\Sigma=\partial D \backslash \Gamma$. In this example, we observe the effect of noise on the numerical solution on $\Sigma$.
Case 1. We choose $d=(0,1)$.

Case 2. We choose $d=(\sqrt{2} / 2, \sqrt{2} / 2)$.

The regularization parameters $\alpha$ chosen by the Morozov discrepancy principle and the errors are given in Tables 1 and 2.

Figures 1, 2, 3, and 4 show the real part of the numerical solutions for different wave numbers with different levels of noise of Cases 1 and 2, respectively.

From the figures and tables, it can be seen that the numerical solutions are stable approximations of the exact solution, and it should be noted that the numerical solution 


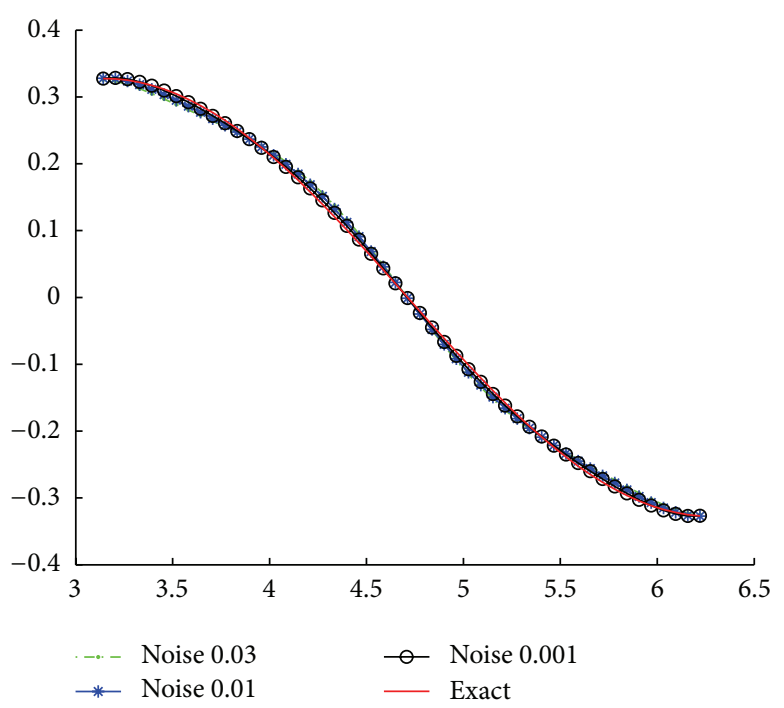

(a) $f$

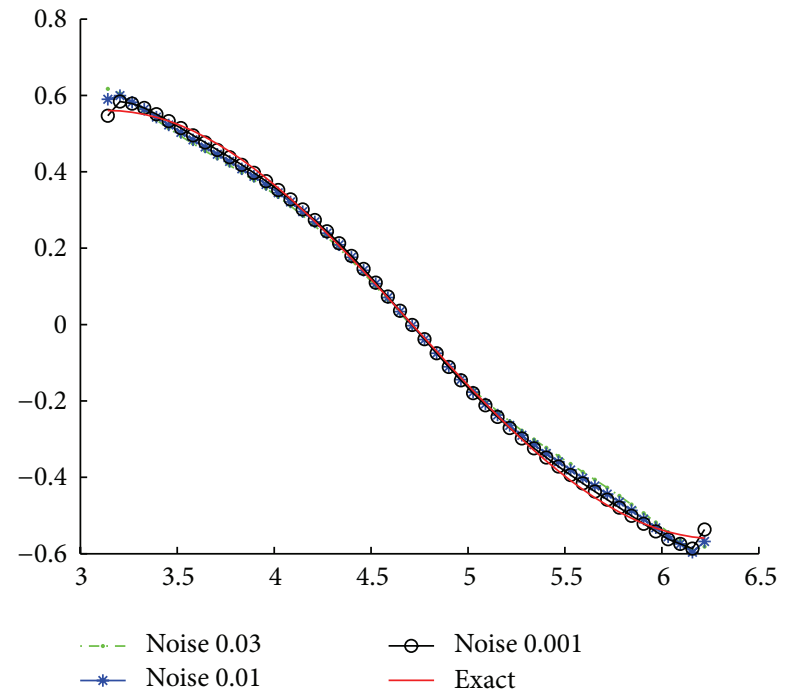

(b) $d$

FIgURE 5: Example 2: the exact solution and the numerical solution on $\Sigma$ with different noises, $k=5$.

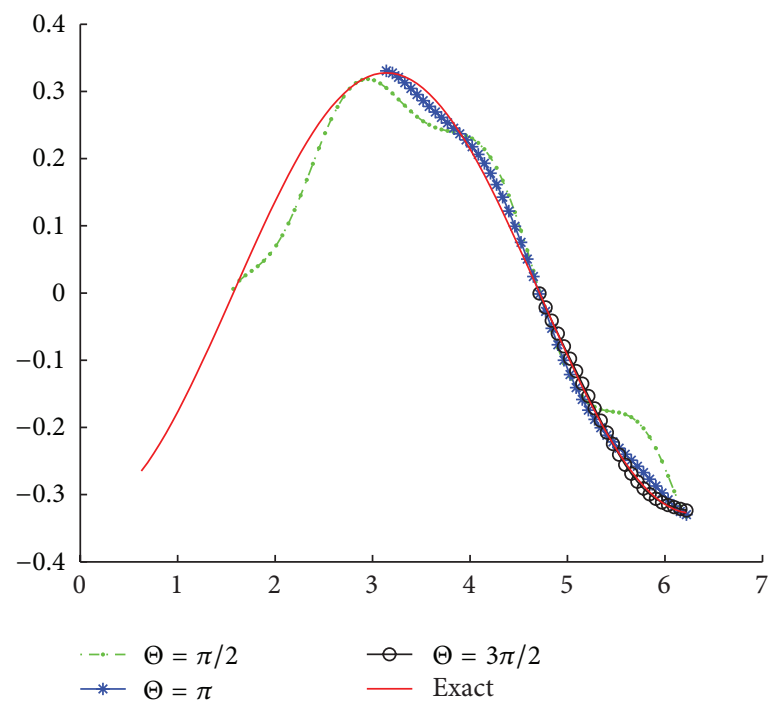

(a) $f$

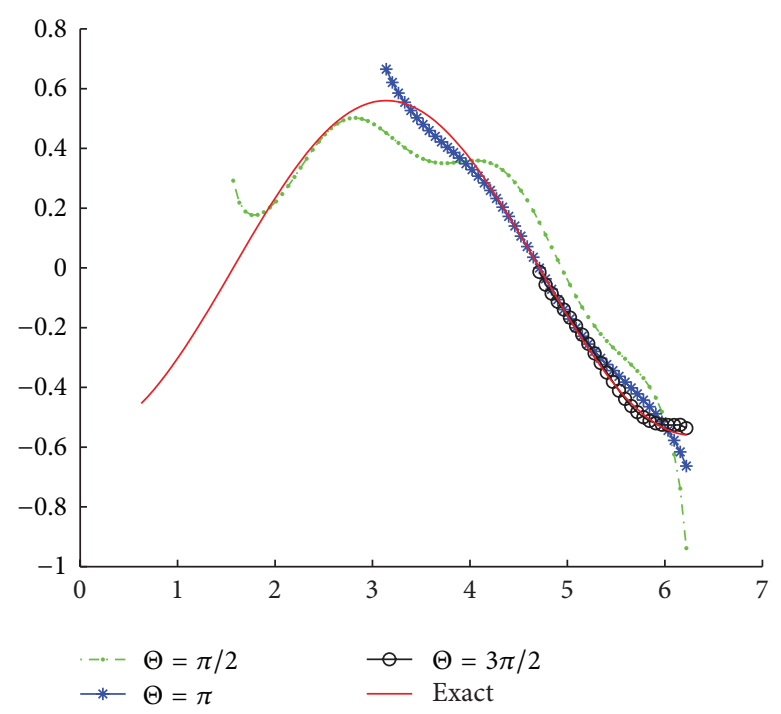

(b) $d$

FIgURE 6: Example 2: the exact solution and the numerical solution on $\Sigma$ with $k=5,1 \%$ noise.

converges to the exact solution as the level of noise decreases.

Example 2. Consider the unit disc $D=\left\{\left(x_{1}, x_{2}\right) \mid x_{1}^{2}+x_{2}^{2}<\right.$ $1\}$. Let $\Gamma=\{x \in \partial D \mid 0<\theta(x)<\Theta\}$ and let $\Sigma=\partial D \backslash \Gamma=\{x \in$ $\partial D \mid \Theta<\theta(x)<2 \pi\}$, where $\theta(x)$ is the polar angle of $x$ and $\Theta$ is a specified angle. In this example, we observe the effect of $\Theta$ on the numerical solution. Choose $u(x)=J_{1}(k r) e^{i \theta}$ as the exact solution, where $J_{1}$ is the Bessel function of order one.

Tables 3 and 4 give the regularization parameters and present the corresponding $L^{2}$ errors and relative $L^{2}$ errors for the approximation of $u$ and $\partial u / \partial n$ on boundary $\Sigma$.
Figure 5 shows the real part of the numerical solution with different levels of noise on $\Theta=\pi$.

In order to investigate the effect of $\Theta$, Figures 6 and 7 show the real part of the numerical solutions with different $\Theta$. It can be seen that large $\Theta$ will improve the results.

\section{Conclusions}

In this paper, we study the application of an integral equations method to solve the Cauchy problem connected with the Helmholtz equation. We give the uniqueness of this problem in Theorem 1, in Section 2, and this cannot be obtained directly since the restriction on $k^{2}$. Then we use the Tikhonov 


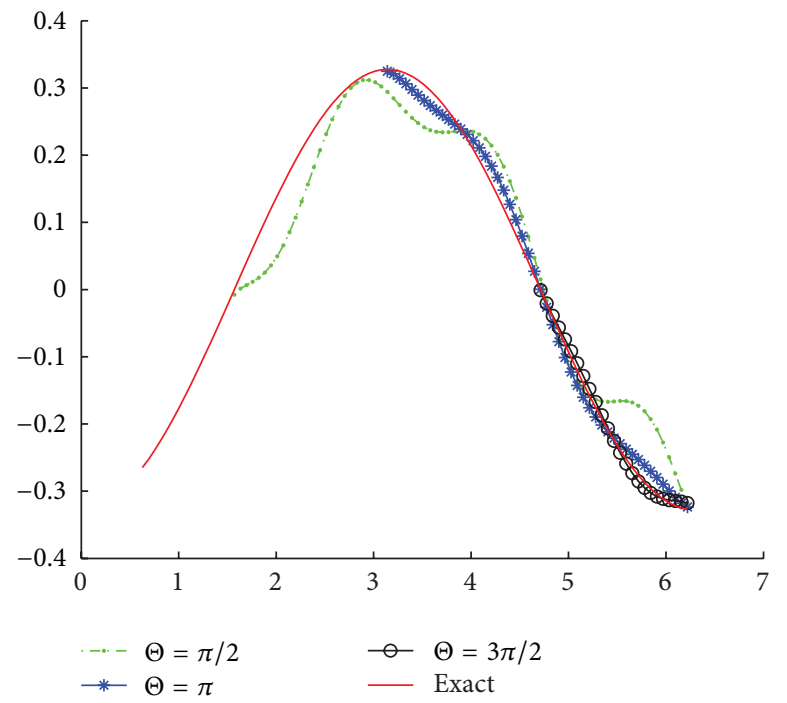

(a) $f$

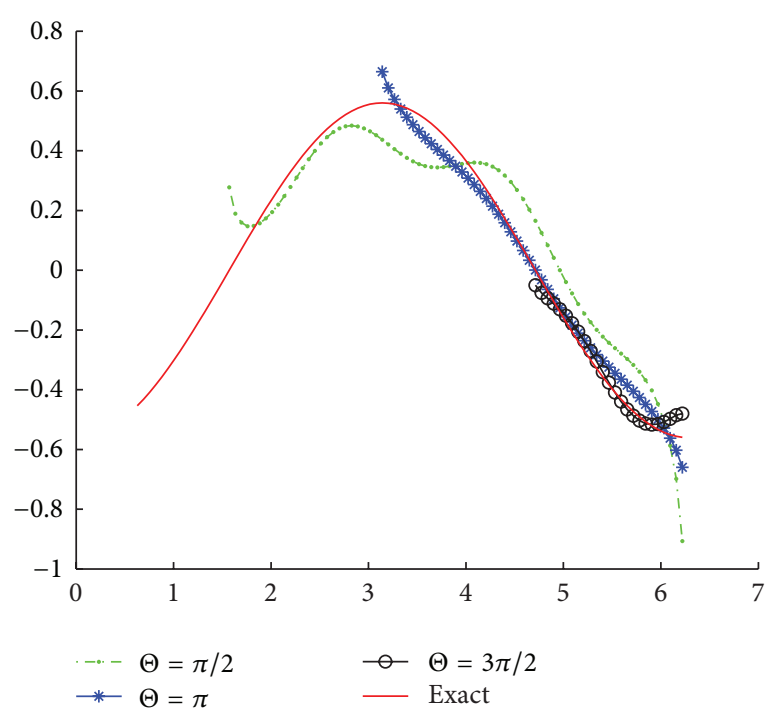

(b) $d$

Figure 7: Example 2: the exact solution and the numerical solution on $\Sigma$ with $k=5,3 \%$ noise.

regularization method with the Morozov discrepancy principle for solving this ill-posed problem. Convergence and stability of the method are then given with two examples. From the examples, we can see that the proposed method is more stable with more Cauchy data, and the numerical results are sensitive about the wavenumber.

\section{Acknowledgments}

The authors would like to thank the editors and the referee for their careful reading and valuable comments which lead to the improvement of the quality of the submitted paper. The research was supported by the NSFC (no. 11201476).

\section{References}

[1] M. R. Bai, "Application of BEM (boundary element method)based acoustic holography to radiation analysis of sound sources with arbitrarily shaped geometries," Journal of the Acoustical Society of America, vol. 92, no. 1, pp. 533-549, 1992.

[2] W. S. Hall and X. Q. Mao, "A boundary element investigation of irregular frequencies in electromagnetic scattering," Engineering Analysis with Boundary Elements, vol. 16, no. 3, pp. 245-252, 1995.

[3] B. K. Kim and J. G. Ih, "On the reconstruction of the vibroacoustic field over the surface enclosing an interior space using the boundary element method," Journal of the Acoustical Society of America, vol. 100, no. 5, pp. 3003-3016, 1996.

[4] L. Marin, L. Elliott, P. J. Heggs, D. B. Ingham, D. Lesnic, and $\mathrm{X}$. Wen, "An alternating iterative algorithm for the Cauchy problem associated to the Helmholtz equation," Computer Methods in Applied Mechanics and Engineering, vol. 192, no. 5-6, pp. 709$722,2003$.

[5] G. Alessandrini, L. Rondi, E. Rosset, and S. Vessella, "The stability for the Cauchy problem for elliptic equations," Inverse Problems, vol. 25, no. 12, Article ID 123004, pp. 1-47, 2009.
[6] T. Hrycak and V. Isakov, "Increased stability in the continuation of solutions to the Helmholtz equation," Inverse Problems, vol. 20, no. 3, pp. 697-712, 2004.

[7] V. Isakov, Inverse Problems for Partial Differential Equations, Springer, New York, NY, USA, 1998.

[8] Y. Sun, D. Zhang, and F. Ma, "A potential function method for the Cauchy problem of elliptic operators," Journal of Mathematical Analysis and Applications, vol. 395, no. 1, pp. 164-174, 2012.

[9] L. Marin, L. Elliott, P. J. Heggs, D. B. Ingham, D. Lesnic, and $\mathrm{X}$. Wen, "Conjugate gradient-boundary element solution to the Cauchy problem for Helmholtz-type equations," Computational Mechanics, vol. 31, no. 3-4, pp. 367-377, 2003.

[10] B. T. Jin and Y. Zheng, "Boundary knot method for the Cauchy problem associated with the inhomogeneous Helmholtz equation," Engineering Analysis with Boundary Elements, vol. 29, no. 10, pp. 925-935, 2005.

[11] B. T. Jin and Y. Zheng, "A meshless method for some inverse problems associated with the Helmholtz equation," Computer Methods in Applied Mechanics and Engineering, vol. 195, no. 1922, pp. 2270-2288, 2006.

[12] L. Marin and D. Lesnic, "The method of fundamental solutions for the Cauchy problem associated with two-dimensional Helmholtz-type equations," Computers \& Structures, vol. 83, no. 4-5, pp. 267-278, 2005.

[13] T. Wei, Y. C. Hon, and L. Ling, "Method of fundamental solutions with regularization techniques for Cauchy problems of elliptic operators," Engineering Analysis with Boundary Elements, vol. 31, no. 4, pp. 373-385, 2007.

[14] T. Wei, H. H. Qin, and R. Shi, "Numerical solution of an inverse 2D Cauchy problem connected with the Helmholtz equation," Inverse Problems, vol. 24, no. 3, Article ID 035003, pp. 1-18, 2008.

[15] W. Chen and Z. J. Fu, "Boundary particle method for inverse cauchy problems of inhomogeneous helmholtz equations," Journal of Marine Science and Technology, vol. 17, no. 3, pp. 157-163, 2009. 
[16] F. Cakoni and R. Kress, "Integral equations for inverse problems in corrosion detection from partial Cauchy data," Inverse Problems and Imaging, vol. 1, no. 2, pp. 229-245, 2007.

[17] F. Cakoni, R. Kress, and C. Schuft, "Integral equations for shape and impedance reconstruction in corrosion detection," Inverse Problems, vol. 26, no. 9, Article ID 095012, 2010.

[18] R. Chapko and B. T. Johansson, "On the numerical solution of a Cauchy problem for the Laplace equation via a direct integral equation approach," Inverse Problems and Imaging, vol. 6, no. 1, pp. 25-38, 2012.

[19] J.-Y. Lee and J.-R. Yoon, "A numerical method for Cauchy problem using singular value decomposition," Korean Mathematical Society, vol. 16, no. 3, pp. 487-508, 2001.

[20] A.-P. Calderón, "Uniqueness in the Cauchy problem for partial differential equations. ," American Journal of Mathematics, vol. 80, pp. 16-36, 1958.

[21] D. Colton and R. Kress, Inverse Acoustic and Electromagnetic Scattering Theory, Springer, New York, NY, USA, 2nd edition, 1998.

[22] W. L. Wendland and E. P. Stephan, "A hypersingular boundary integral method for two-dimensional screen and crack problems," Archive for Rational Mechanics and Analysis, vol. 112, no. 4, pp. 363-390, 1990.

[23] R. Kress, "Inverse scattering from an open arc," Mathematical Methods in the Applied Sciences, vol. 18, no. 4, pp. 267-293, 1995.

[24] M. Durand, "Layer potentials and boundary value problems for the Helmholtz equation in the complement of a thin obstacle," Mathematical Methods in the Applied Sciences, vol. 5, no. 3, pp. 389-421, 1983.

[25] A. Kirsch, An Introduction to the Mathematical Theory of Inverse Problems, Springer, New York, NY, USA, 1996.

[26] D. Colton and R. Kress, Integral Equation Methods in Scattering Theory, Wiley-Interscience, New York, NY, USA, 1983. 


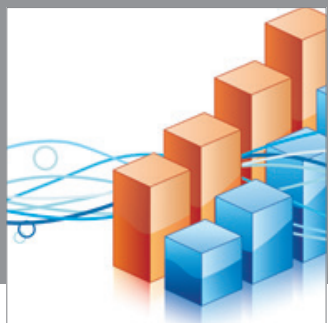

Advances in

Operations Research

mansans

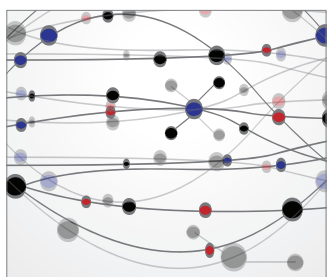

The Scientific World Journal
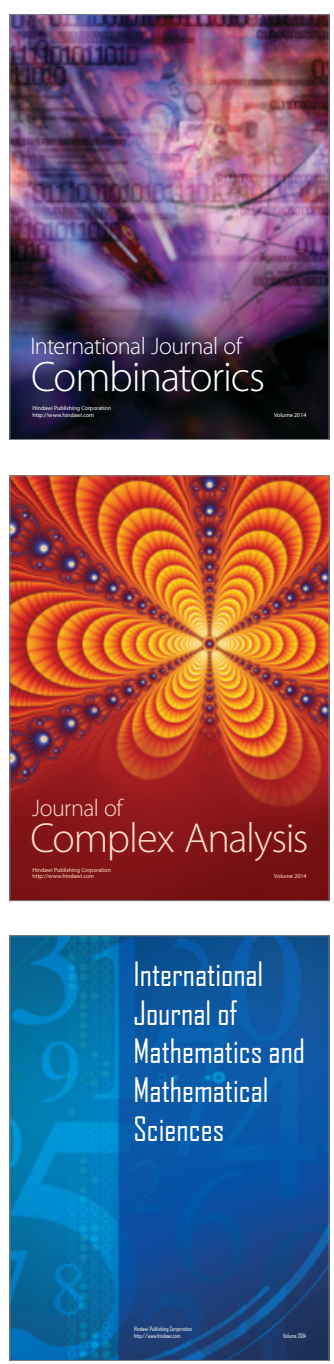
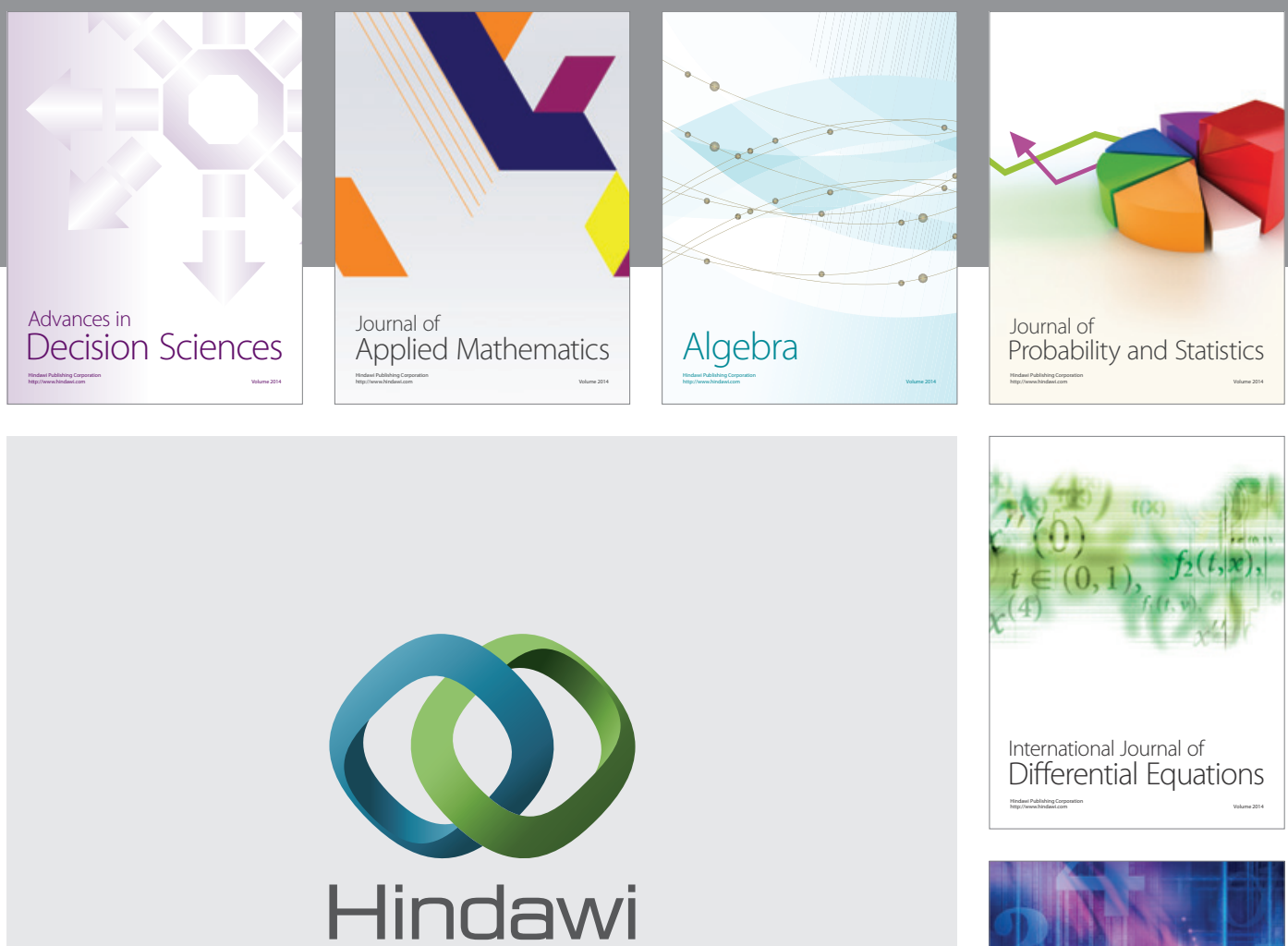

Submit your manuscripts at http://www.hindawi.com
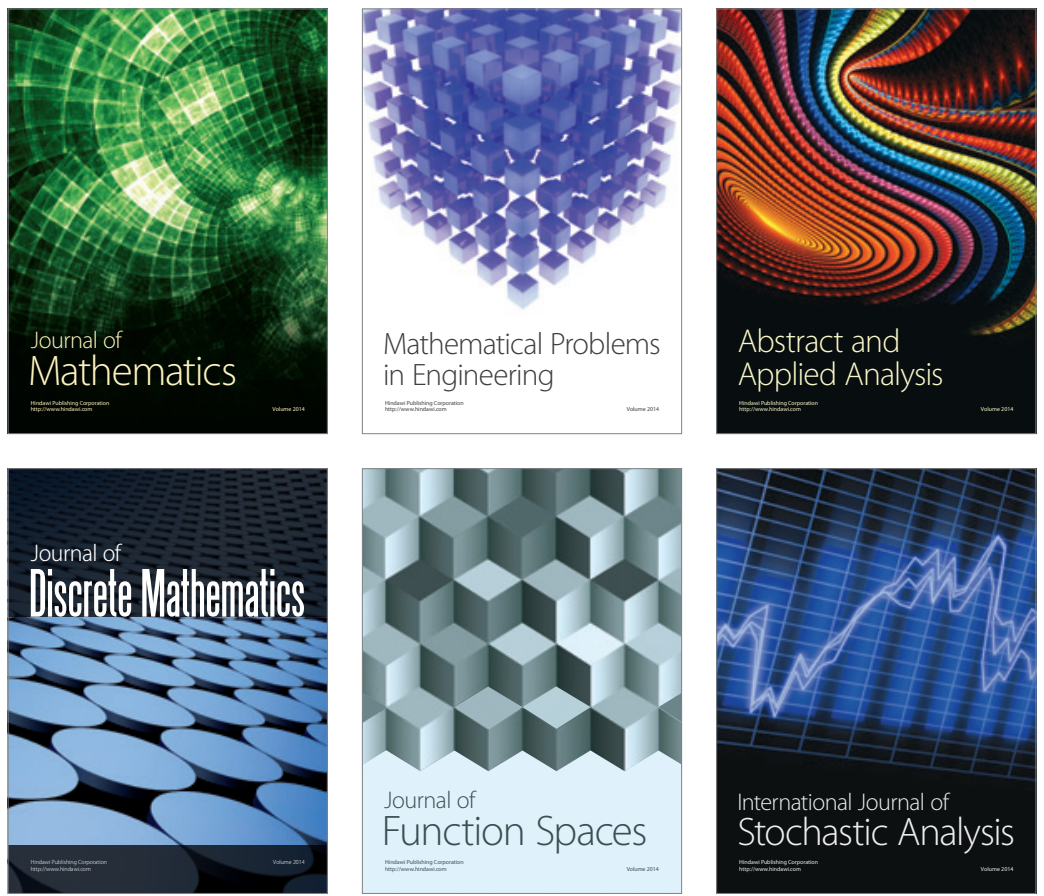

Journal of

Function Spaces

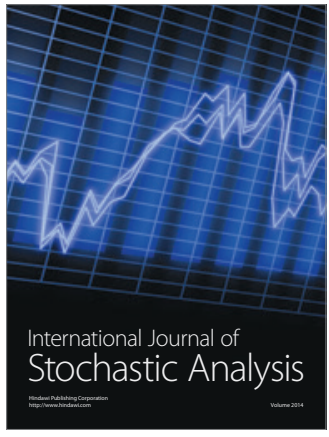

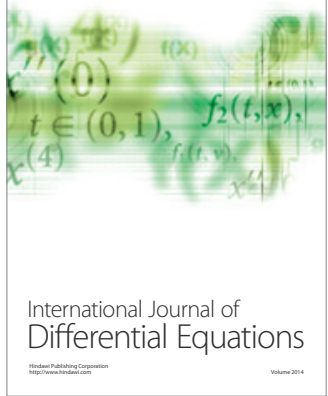
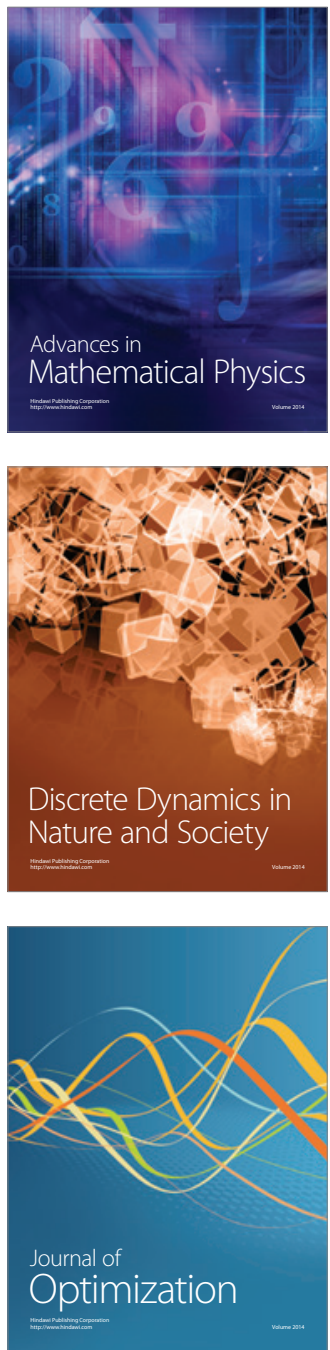